\title{
Life Quality Increase Using Locomotion in Students with Disabilities
}

\author{
K.V. Davletjarova ${ }^{1, a}$, L.V. Kapilevich ${ }^{1,2}$, N.A. Ovchinnikova ${ }^{1}$ \\ ${ }^{1}$ Tomsk Polytechnic University, 634050 Lenin Avenue, 30, Tomsk, Russia \\ ${ }^{2}$ Tomsk State University, 634050 Lenin Avenue, 36, Tomsk, Russia
}

\begin{abstract}
The aspects of the bioelectrical muscular activity of lower extremities, motor coordination and balance in football players with musculoskeletal disorders when performing a ball shot have been studied. The study has demonstrated that football players with musculoskeletal disorders while performing a ball shot experienced movement linearity disorders, manifested as motor coordination disorders, possibly related to the displacement of the general centre of gravity. However, these disorders in student football players were compensated by decrease of the general centre of gravity range and the fluctuation rate in sagittal and frontal planes, thus promoting an increase of the balance quality. The student football players with musculoskeletal disorders while performing a ball shot experienced simultaneous restructure in the activity of lower extremity muscles: an increase of lower leg muscle activity was accompanied by a hip muscle activity decrease. The results would help us to correct the training program for football players with musculoskeletal disorders, by adding physical exercises, aimed at strengthening hip muscles and the inclusion of additional coordination and balance exercises.
\end{abstract}

\section{Introduction}

Musculoskeletal system disorders (MSD) is one the main reasons, decreasing physical activity of high school students. Balance and coordination disorders are considered as one of the manifestations, associated with this pathology [1,4]. Students, affected by these disorders, cannot participate in many leisure activities, popular in youngsters; this results in partial isolation from their social group [3].

Nowadays, modern students with virtually no health issues are extremely low in numbers. The majority of students, playing football, suffer from musculoskeletal system pathology (MSD): scoliosis, osteochondropathy, and osteochondrosis in remission phase, planus. It is associated with the fact that regardless of health disorders young people tend to reject any restriction in socialisation and sports either.

The laws of biomechanics are used in modern sports to study biomechanical aspects of the musculoskeletal system. The biomechanic science studies positioning laws of human body within normal parameters and in pathology, related to standing, sitting, running, walking (posture, location of gravity centre, balance area estimation, joint closure methods and accommodation processes for keeping the centre of gravity within the balance area; principles of choice for medical (preventive) measures with account of biomechanical unity of MSD.

Youth involvement with popular sports is one of the ways to solve this problem. Besides, when developing training programs, the coaches should consider health aspects and a motor ability rate in students $[2,5]$. Understanding of physiological mechanisms, ensuring compensation of motor disorders in young people, affected by MSD, is necessary to build a balanced training system.

Football is a difficult kind of sport not only in physical but also psychological aspects. When engaging in football activities, an individual develops dexterity, quickness, motor reactions, space orientation and movement coordination. Besides, ball games promote the development of know-how and skills in a team, educate mutual cooperation. They demand emotional balance, braveness and good physical condition of every athlete.

Thus, when preparing training program, one should consider motor ability aspects and individual mobility. Incorrect use of training methods, compromising laws of motor biomechanics, may lead to the occurrence of muscular imbalance, worsening pre-existing MSD pathologies.

Aim of this work: to study the aspects of the bioelectrical muscular activity of lower extremities,

${ }^{a}$ Corresponding author: davletyarova@rambler.ru 
motor coordination and balance in football players with musculoskeletal disorders when performing a ball shot.

\section{Materials and methods}

The study subjects were I-II year students $(n=30)$ of Tomsk Polytechnic University, 18-20 years of age, with specialised training in "football". We have formed two groups. First (main) group consisted of students $(n=15)$ with MSD (II-III grade scoliosis secondary to II-III grade planus). Second (control) group $(n=15)$ involved students without MSD pathologies. Intensity, the regular basis of training and the training rate were equal in both groups.

Bioelectrical activity assessment of right leg muscles, exposed to performing a ball shot, was conducted using the multi-functional computer electroneuromyography device "Neuro - MVP-4", consisting of a subject unit with a modificationdependent set of channels, educational software and documentation. The complex helps to conduct a fourchannel electrical activity study of muscles and nervous system, as well as somatosensory, ocular and auditory brain potential evoked responses and biopotentials during magnetic stimulation. The study was conducted using surface electrodes, appearing as metal disks with the area up to $1 \mathrm{~cm}^{2}$. The students were offered to perform a ball shot using the middle part of foot lift. The electrodes were applied according to anatomic muscle location:

1) m. gastrocnemius, caput laterale;

2) m. gastrocnemius, caput media;

3) m. vastus lateralis;

Interferential EMG allows reading information about muscle condition, predicting a lesion rate, including extrapyramidal pathology. The essence of this method is about registration of bioelectrical activity of the muscle using epicutaneous electrodes.

To assess motor coordination when performing a ball shot, we used posturography (stabilography) analyser Stabilan-1 (CJSC "Experimental Design Office "RHYTHM", Taganrog). It is a complex of technical and educational software and documentation based on computerized posturography, used for diagnosis of human body balance disorders, restoration of motor and coordination disorders. To study aspects of motor skills and balance parameters in football players with MSD when performing a ball shot, we used posturography test.

During the study we assessed and registered the following parameters:

- Xcp - initial displacement of the centre of pressure (CoP) in frontal (left to right) plane, $\mathrm{mm}$

- Ycp - initial displacement of the centre of pressure in sagittal direction (forth and back), $\mathrm{mm}$

- Qx - dispersion (deviation rate) of the centre of pressure in frontal plane, $\mathrm{mm}$

- Qy - dispersion (deviation rate) of the centre of pressure in sagittal plane, $\mathrm{mm}$

- Medium displacement rate of the centre of pressure, $\mathrm{mm} / \mathrm{s}$.
- Ellipse area, sq. mm.

- Time rate of statokinetic profile, $\mathrm{sq} \mathrm{mm} / \mathrm{s}$

- Equilibrium rate quality (ERQ), \%

- Medium linear rate, $\mathrm{mm} / \mathrm{s}$.

The football players performed a ball shot using the middle part of foot lift while standing on the posturography platform (force plate). The actual data are presented as "medium \pm error in mean" $(\mathrm{M} \pm \mathrm{m})$. The significance of the difference between groups was estimated using Mann-Whitney U test.

\section{Results and discussion}

A statokinetic profile of performing ball shots by football players in main and control group are presented in Figure 1. The football players in the preparatory phase clearly demonstrated fluctuations of the centre of pressure $(\mathrm{CoP})$. CoP significantly deviated back before the performance of the shot, trajectory of movement in the shot phase was distorted. Following the shot in the sagittal plane, pronounced deviations of CoP were reported.

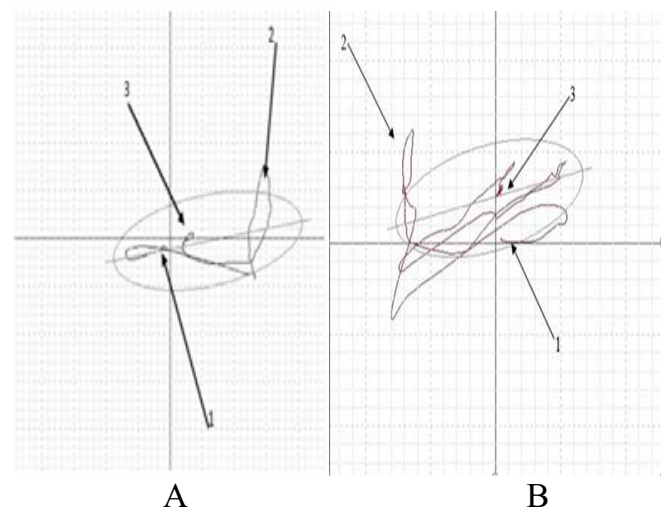

Fig. 1. The statokinetic profile of performing a shot by a football player in the control group (A) and a football player with MSD (B)

Note: arrows point to the following:

1. Start of the movement

2. Moment of performing a ball shot

3. End of the movement.

To the contrary, the football players in the control group in the preparatory phase experienced displacement of $\mathrm{CoP}$ to the right. Before the shot, $\mathrm{CoP}$ shifted back, but with a lesser range than that in the main group. The movement trajectory of $\mathrm{CoP}$ when performing a ball shot was devoid of fluctuations and almost straight. After performing a ball shot, CoP shifted to the left.

Table 1 represents main parameters of motor coordination when performing ball shots by football players from both groups (Figure $1 \mathrm{~A}, \mathrm{~B}$ ). 
Table 1. Posturography parameters when performing a ball shot

$(\mathrm{X} \pm \mathrm{m})$

\begin{tabular}{|l|l|l|}
\hline Parameters & Main group & Control group \\
\hline $\begin{array}{l}\text { Dispersions in } \\
\text { frontal line, mm }\end{array}$ & $34.8 \pm 2.6^{*}$ & $45.9 \pm 2.2$ \\
\hline $\begin{array}{l}\text { Dispersions in } \\
\text { sagittal line, mm }\end{array}$ & $18.5 \pm 1.5^{*}$ & $29.0 \pm 4.4$ \\
\hline $\begin{array}{l}\text { Medium } \\
\text { displacement rate of } \\
\text { CoP, mm/s }\end{array}$ & $112.5 \pm 9,2^{*}$ & $141.8 \pm 6.2$ \\
\hline $\begin{array}{l}\text { Ellipse area, sq. mm. } \\
\text { Equilibrium rate } \\
\text { quality (ERQ), \%; }\end{array}$ & $7879.7 \pm 1101,5^{*}$ & $13005.8 \pm 1468.1$ \\
\hline $\begin{array}{l}\text { Medium linear rate, } \\
\text { mm/s. }\end{array}$ & $113.3 \pm 9.3^{*}$ & $11.4 \pm 1.5$ \\
\hline
\end{tabular}

* - significance in contrast to the control group, $\mathrm{p}<0.05$

In the control group of students, dispersion rates in frontal and sagittal planes were significantly $(p<0.05)$ higher, than in the group of football players with MSD.

Also, when compared to the main group, parameters of the medium displacement rate of $\mathrm{CoP}$, ellipse area and the medium linear rate were significantly $(p<0.05)$ higher. However, the equilibrium rate quality (ERQ) in the main group was significantly $(\mathrm{p}<0.05)$ higher, than in the control group (Table 1).

Thus, the football players with MSD experienced coordination disorders, leading to movement linearity disorder, associated with displacement of the general centre of gravity of the body (CoG). Besides, when performing shots, CoP trajectory was distorted in all phases. We demonstrated that athletes compensated these disorders by a decrease of the following parameters, such as dispersion in frontal and sagittal planes, the medium linear rate, ellipsis area, the medium displacement rate of the centre of pressure. Also, we reported an increase of the balance function quality in the main group.

In Table 2 bioelectrical muscular activity of the lower extremities when performing a ball shot using the middle part of foot lift in main and control groups is presented. In football players with MSD, we observed a significant $(\mathrm{p}<0.05)$ increase of the maximal range of bioelectrical activity of lower leg muscles: m.gastrocnemius, caput laterale by $21 \%$ and m. gastrocnemius, caput mediale by $24 \%$. However it was demonstrated, that the bioelectrical activity rate for $\mathrm{m}$. vastus lateralis and $\mathrm{m}$. rectus femoris was significantly $(\mathrm{p}<0.05)$ lower, that in healthy football players (Figure 2).

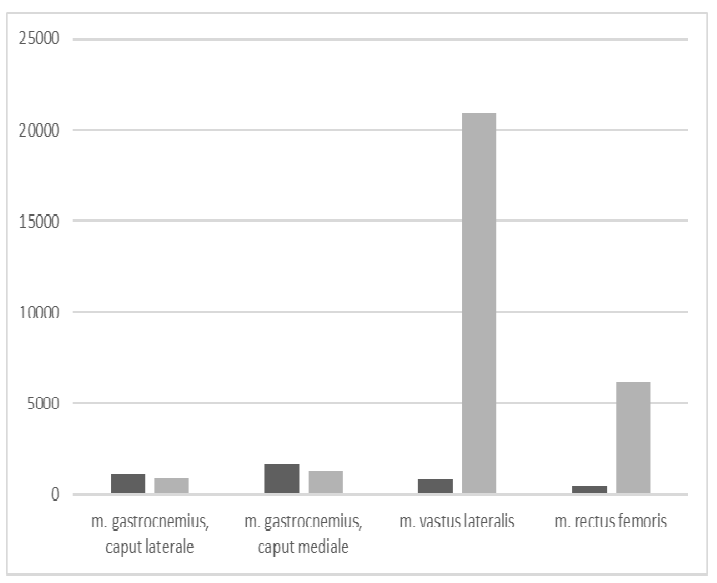

Fig. 2. The maximum range of bioelectrical muscular activity of the lower extremities when performing a ball shot using the middle part of foot lift

Note:

Black colour - main group;

Grey colour - control group

* - significance in contrast to the control group, $\mathrm{p}<0.05$

Thus, football players with MSD, when performing a ball shot using the middle part of foot lift, mostly involved lower leg muscles (gastrocnemius, caput laterale and $\mathrm{m}$. gastrocnemius, caput mediale), when football players without MSD pathology performed a shot mostly owing to hip muscle activity (m. vastus lateralis, $\mathrm{m}$. rectus femoris) (Table 2).

Table 2. The maximum rate of bioelectrical muscular activity of lower extremities when performing a ball shot

$(\mathrm{X} \pm \mathrm{m})$

\begin{tabular}{|c|c|c|}
\hline Group & Main group & Control group \\
\hline $\begin{array}{l}\text { m. gastrocnemius, } \\
\text { caput laterale }\end{array}$ & $1120 \pm 354.3 *$ & $874.1 \pm 255.1$ \\
\hline $\begin{array}{l}\text { m. gastrocnemius, } \\
\text { caput mediale }\end{array}$ & $\begin{array}{l}1680.1 \pm 660.6 \\
*\end{array}$ & $1280 \pm 345.9$ \\
\hline m. vastus lateralis & $801.7 \pm 345.6^{*}$ & $\begin{array}{l}20904 \pm 17111 \\
3\end{array}$ \\
\hline m. rectus femoris & $513.3 \pm 230.9^{*}$ & $6180 \pm 3824.6$ \\
\hline
\end{tabular}

Therefore, we have demonstrated that football players with MSD when performing a ball shot experienced strain redistribution in lower extremity muscles. Along with the decrease in hip muscle activity, lower leg muscles were involved in locomotion.

\section{Conclusion}

Football players with musculoskeletal disorders while performing a ball shot experienced movement linearity 
disorders, manifested as motor coordination disorders, possibly related to the displacement of the general centre of gravity. However, these disorders in student football players were compensated by the decrease of the general centre of the gravity range and the fluctuation rate in sagittal and frontal planes, thus promoting the increase of balance quality.

The student football players with musculoskeletal disorders while performing a ball shot experienced simultaneous restructure in the activity of lower extremity muscles: increase of lower leg muscle activity was accompanied by a hip muscle activity decrease. It is associated with compensatory processes: increase of muscular activity in distal compartments of the extremity, correcting more successfully the deviations of the CoG trajectory from the normal value.

The results would help us to correct the training program for football players with musculoskeletal disorders, by adding physical exercises, aimed at strengthening hip muscles and the inclusion of additional coordination and balance exercises.

\section{Acknowledgment}

This research was conducted owing to the grant, issued by the Russian Science Foundation (Project No. 16-1800016).

\section{References}

1. V.I. Dubrovskyy, V.N. Fedorova, Pathological biomechanics (Publishing house VLADOS PRESS, 2003)

2. A.V. Illarionova, L.V. Kapilevich, Theory and practice of physical culture, 12 (2014)

3. E.V. Koshelskaja, L.V. Kapilevich, V.N. Bajenov, V.I. Andreev, O.I. Buravel. Bulletin of Experimental Biology and Medicine, 153, 2 (2012)

4. M.S. Nagornov, K.V. Davletjyarova, A.A. Iljin, L.V. Kapilevich, Teoriya i Praktika Fizicheskoy Kultury, 7, (2015)

5. M.E. Geisser, The Journal of Pain, 6(11), (2005) 